\title{
Why do house-hunting ants recruit in both directions?
}

\author{
R. Planqué - F.-X. Dechaume-Moncharmont • \\ N. R. Franks • T. Kovacs • J. A. R. Marshall
}

Received: 12 February 2007 / Revised: 21 March 2007 / Accepted: 20 May 2007 / Published online: 3 August 2007

(C) Springer-Verlag 2007

\begin{abstract}
To perform tasks, organisms often use multiple procedures. Explaining the breadth of such behavioural repertoires is not always straightforward. During house hunting, colonies of Temnothorax albipennis ants use a range of behaviours to organise their emigrations. In particular, the ants use tandem running to recruit naïve ants to potential nest sites. Initially, they use forward tandem runs (FTRs) in which one leader takes a single follower along the route from the old nest to the new one. Later, they use reverse tandem runs (RTRs) in the opposite direction. Tandem runs are used to teach active ants the route between the nests, so that they can be involved quickly in nest evaluation and subsequent recruitment. When a quorum of decisionmakers at the new nest is reached, they switch to carrying nestmates. This is three times faster than tandem running. As a rule, having more FTRs early should thus mean faster emigrations, thereby reducing the colony's vulnerability. So why do ants use RTRs, which are both
\end{abstract}

\section{R. Planqué $(\varangle)$}

Department of Mathematics, VU University Amsterdam, De Boelelaan 1081a, 1081 HV Amsterdam, The Netherlands e-mail: rplanque@few.vu.nl

F.-X. Dechaume-Moncharmont

Equipe Écologie Évolutive, UMR CNRS 5561

BioGéoSciences, Université de Bourgogne, 6 Bd Gabriel,

21000 Dijon, France

N. R. Franks

School of Biological Sciences, University of Bristol,

Woodland Road, Bristol BS8 1UG, UK

T. Kovacs $\cdot$ J. A. R. Marshall

Department of Computer Science, University of Bristol,

Woodland Road, Bristol BS8 1UB, UK slow and late? It would seem quicker and simpler for the ants to use more FTRs (and higher quorums) to have enough knowledgeable ants to do all the carrying. In this study, we present the first testable theoretical explanation for the role of RTRs. We set out to find the theoretically fastest emigration strategy for a set of emigration conditions. We conclude that RTRs can have a positive effect on emigration speed if FTRs are limited. In these cases, low quorums together with lots of reverse tandem running give the fastest emigration.

Keywords Recruitment methods - Social insects • Tandem running - Temnothorax albipennis • Collective behaviour

\section{Introduction}

Organisms often employ more than one mechanism to accomplish a task. For instance, animals typically navigate with multiple 'input channels'. The classic example is homing by the rock dove Columba livia, for which magnetic fields, the sun, landmarks and geophysical processes have all been shown to be used (Wiltschko and Wiltschko 2003).

The range of behaviours or mechanisms organisms employ may be puzzling. At times, an apparent simplicity is observed. Defence strategies against predators are a well-studied example here. Acacia trees (Acacia spp.) either have chemical defences to ward off herbivores or have symbiotic relationships with protective ants (Rehr et al. 1973). Hosts parasitised by Eurasian cuckoos Cuculus canorus famously reject cuckoo eggs, but never reject cuckoo chicks (Davies 2000). This simplicity may be the result of evolutionary lag but, more interestingly, 
may also be caused by one strategy making another maladaptive by reducing predator abundance (Planqué et al. 2002; Britton et al. 2007).

Conversely, the portfolio of behaviours may be varied and complex. Different mechanisms may complement one another, and true redundancy is often hard to show (Able and Bingman 1987). Indeed, the existence of a suite of mechanisms against a broad ensemble of predators is readily understandable. One exemplar is the vertebrate immune system (Perelson 2002).

Another striking example of a system in which different mechanisms augment and complement one another, but now at a collective level, is house hunting in social insects. This has become one of the model systems to study distributed decision making in animals. When the nest is destroyed, the colony has to decide collectively where to settle next during a time of crisis (Franks et al. 2003a). Individual ants or bees have been shown to combine sophisticated assessments of potential nest sites (Seeley 1977; Seeley and Morse 1978; Mallon and Franks 2000; Franks et al. 2003b) with various recruitment mechanisms to collate information, and thus make collective decisions (Mallon et al. 2001; Pratt et al. 2002; Pratt 2005; Seeley and Visscher 2003, 2004; Visscher 2007).

A typical emigration by a colony of Temnothorax albipennis may be described as follows. When the old nest is destroyed, a fraction of ants goes out scouting to find a new home. Upon finding a nest, the nest is assessed (Mallon and Franks 2000) and ants start recruiting other ants to it with a latency that is inversely proportional to the perceived nest quality (Mallon et al. 2001), using a process called forward tandem running (Möglich et al. 1974). During a forward tandem run, a knowledgeable ant teams up with a naïve ant. The leader slowly progresses towards the new nest, each time waiting for the follower to catch up, thereby teaching her the way (Franks and Richardson 2006). Through this slow recruitment process, information on the location of the new nest spreads, and recruiter numbers increase. Once a nest population reaches a certain quorum threshold, the recruiters switch from slow tandem running to much faster social carrying, and transport the remaining passive ants and brood to the new nests (Pratt et al. 2002; Pratt 2005).

This description has been the basis of several models (Pratt et al. 2002, 2005; Marshall et al. 2006; Planqué et al. 2006). However, a behaviour commonly employed by these ants is usually not included (but see Pratt et al. 2005) and has never been analysed. After the quorum, recruiter ants are not only engaged in social carrying, but also regularly perform tandem runs from the new back to the old nest. These so-called reverse tandem runs (Möglich 1978) are often more common than forward tandem runs (Mallon et al. 2001; Pratt et al. 2002), but their function is much less well understood.

To maximise fitness, the colony should emigrate as quickly as possible to avoid predation and other hazards. Therefore, during house hunting, a fast build up of recruiters is essential. Why then do ants mix fast carrying with slow reverse tandem running, when they already have forward tandem running at their disposal?

In this paper, the role of reverse tandem running is theoretically investigated. In particular, through the use of mathematical models, we explore under what circumstances reverse tandem running can have a positive influence on emigration speed.

\section{Materials and methods}

We present two mathematical models to investigate the possible role of reverse tandem runs in ant colony emigrations. Reverse tandem running does not contribute to the decision-making process of which new nest to choose (Pratt et al. 2002; Franks et al. 2003a). We, therefore, restrict ourselves to emigrations to one new nest only.

We model the emigration as follows (Fig. 1). Only a small fraction of the ants in a colony are actively involved during emigrations (Pratt et al. 2002; Langridge 2006). In this paper, we thus divide the colony's $N$ ants into fractions $F N$ of active ants $A$, and $(1-F) N$ passive ants $P$. This assumption to divide ants into active and passive is a crucial one, without which the models collapse. We will come back to this in "Discussion". Numbers of scouts and recruiters are denoted $S$ and $R$, respectively. The rates at which active ants leave the nest and become scouts, and scouts recruiters, are given by $\mu$ and $k$, respectively. Forward tandem running occurs at a rate $\lambda$ until the quorum $Q$ is met, after which recruiters carry passive ants and brood at a rate $\phi$. To incorporate reverse tandem runs, we need to model which ants follow these tandem runs. Available data from nest-choice experiments (Mallon et al. 2001) suggest two possibilities: Either the reverse tandem runs are followed by ants that have not found the new nest yet, or by ants that have been carried to the new nest. These two options are not necessarily mutually exclusive: the carried ants could have been scouts. We modelled both these two possible interpretations. Model 1 assumes that reverse tandem runs are followed by uncommitted scouts in the arena, and model 2 assumes they are followed by passive ants that were carried to the new nest. 

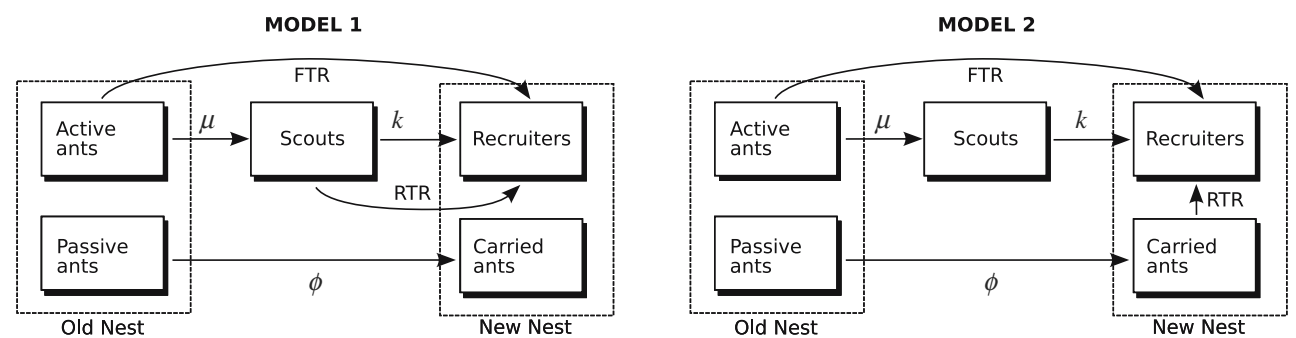

Fig. 1 Diagrams of the two different models for which reverse tandem runs are hypothesised to increase speed. FTR Recruitment through forward tandem running; $R T R$ recruitment through reverse tandem running. The parameters are explained in Table 1

To capture in detail the influence of reverse tandem runs on emigration dynamics, we need to consider the following points:

1. Both tandem running and social carrying involve a pair of ants from two different classes. Hence, recruitment can only occur if ants of both participating classes are available;

2. Once the quorum has been met, recruiters cannot carry and perform reverse tandem runs simultaneously (we also assume recruiters are not involved in other activities than these two).

The interaction between different classes of ants has been modelled using simple interaction terms. We assume that ants of both classes are well mixed in the part of the arena (or nest) where they meet. With populations of ants of size $X$ and $Y$ meeting, the number of ants that on average meet is then proportional to $X Y /(X+Y)$. Importantly, the smallest class limits the interaction rate, as is to be expected.

We thus also have to specify how much post-quorum time recruiters spend on carrying or reverse tandem running (they are assumed not to spend any time on other behaviours). Before the quorum is met, the rate at which active ants at the old nest, $A$, become recruiters, $R$, through tandem running is given by $\lambda R A /(A+R)$. Now let $f$ be the fraction of postquorum time spent on reverse tandem runs, and the remainder $1-f$ spent on social carrying. Then the mean number of scouts becoming recruiters through reverse tandem runs is

$f \lambda R S /(S+R)$.

For carried ants becoming recruiters through reverse tandem running, we have by analogy

$f \lambda R C /(C+R)$.

Similarly, the mean number of passive ants $P$ that become carried ants is given by $(1-f) \phi R P /(P+R)$. Recruiter ants should not perform reverse tandem runs when there are no scouts or carried ants left. Therefore, we replace $f$ by $\bar{f}$ in Eqs. 1 and 2, where $\bar{f}=$ $\min \{S f, f\}$ and $\min \{C f, f\}$, respectively. The $\min$ operation is for computational reasons only and ensures that $\bar{f}$ decreases continuously but rapidly to zero as $S$ or $C$ decreases, respectively. It has no influence on the models' predictions. We drop the bar on $\bar{f}$ in the rest of the paper.

Forward tandem running only occurs before the quorum is met and carrying and reverse tandem running

Table 1 Values or ranges, where applicable, for the parameters used in models 1 and 2 depicted in Fig. 1

\begin{tabular}{lll}
\hline Parameters & Description & Value/range \\
\hline$N$ & Colony size & 250 \\
$F$ & Fraction of active ants & {$[0.05,0.5]$} \\
$Q$ & Quorum threshold & n.a. \\
$f$ & Fraction of post-quorum reverse tandem running time & n.a. \\
$\mu$ & Rate at which active ants at old nest become scouts $\left(\mathrm{ant}^{-1} \min ^{-1}\right)$ & {$[0.01,0.2]$} \\
$\lambda$ & Rate at which ants following tandem runs become recruiters $\left(\operatorname{ant}^{-1} \min ^{-1}\right)$ & 0.1 \\
$\phi$ & Rate at which passive ants are carried to new nest $\left(\operatorname{ant}^{-1} \min ^{-1}\right)$ & 0.2 \\
$k$ & Rate at which scouts independently become recruiters $\left(\mathrm{ant}^{-1} \min ^{-1}\right)$ & $\{0.0001,0.001\}$ \\
\hline
\end{tabular}

Parameter choices for $\lambda, \phi$ and $N$ were taken from the ranges in Pratt et al. (2005). 
only after. These are modelled with functions $l, c$ and $r$, respectively, as follows.

$$
\begin{aligned}
& l(\lambda, R, Q, A)= \begin{cases}\lambda \frac{R A}{R+A} & \text { if } R<Q, \\
0 & \text { otherwise }\end{cases} \\
& c(\phi, R, Q, P)= \begin{cases}\phi \frac{R P}{R+P} & \text { if } R \geq Q, \\
0 & \text { otherwise }\end{cases}
\end{aligned}
$$

and, setting $B$ to $A$ for model 1 , and to $P$ for model 2,

$r(\lambda, R, Q, B)= \begin{cases}\lambda \frac{R B}{R+B} & \text { if } R \geq Q \\ 0 & \text { otherwise }\end{cases}$

To aid the reader, we state the full equations for both models. The equations for model 1 are given by

$$
\left\{\begin{array}{l}
\dot{A}=-\mu A-l(\lambda, R, Q, A) \\
\dot{S}=\mu A-k S-f r(\lambda, R, Q, S) \\
\dot{R}=k S+l(\lambda, R, Q, A)+f r(\lambda, R, Q, S) \\
\dot{P}=-(1-f) c(\phi, R, Q, P) \\
\dot{C}=(1-f) c(\phi, R, Q, P)
\end{array}\right.
$$

with initial conditions $(A, S, R, P, C)(0)=(F N-2 \varepsilon$, $\varepsilon, \varepsilon,(1-F) N, 0)$. Model 2 is specified by

$$
\left\{\begin{array}{l}
\dot{A}=-\mu A-l(\lambda, R, Q, A) \\
\dot{S}=\mu A-k S \\
\dot{R}=k S+l(\lambda, R, Q, A)+f r(\lambda, R, Q, C), \\
\dot{P}=-(1-f) c(\phi, R, Q, P) \\
\dot{C}=(1-f) c(\phi, R, Q, P)-f r(\lambda, R, Q, C)
\end{array}\right.
$$

with the same initial conditions as model 1. Models 1 and 2 only differ in the placement of the term $\operatorname{fr}(\lambda, R, Q, C)$. In both models, the $\varepsilon$ in the initial conditions is necessary to avoid singularities in the denominators of the interaction functions $l, r$ and $c$. We have used $\varepsilon=0.01$ throughout.

We also explored a number of other models in which some assumptions were relaxed. These are briefly discussed in the final section of this paper.

The main hypothesis we tested on both these models was: Reverse tandem runs speed up the emigration if recruiter numbers failed to increase early in the emigration; this occurs through a combination of the new nest being hard to find and forward tandem running being prohibited. We tested this hypothesis by finding the fastest emigration strategy for given parameter settings and determining whether reverse tandem runs formed part of this optimal strategy. We first fixed the scouting parameter, $\mu$, the fraction of active ants at the old nest, $F$, and $k$, the rate at which scouts become recruiters. Then quorum size $Q$ and fraction of post-quorum reverse tandem running time $f$ were varied to optimise emigration speed. Individual runs were performed in Matlab using a standard ode45 solver. Emigrations were termed completed when all passive ants and active ants at the old nest had disappeared. In particular, we set the threshold for emigration completeness at $P+A=0.01$. Optimal strategies were found using the Nelder-Mead simplex method (Nelder and Mead 1965). Parameters $\mu$ and $F$ were varied on an equidistant $20 \times 20$ grid spanning [0.01, 0.2] $\times[0.05,0.5]$. Recruitment latency $k$ was set at either 0.0001 or 0.001 . The ranges of these parameters are inspired by experimental estimates in (Pratt et al. 2002; Pratt 2005). Parameter values are summerised in Table 1.

\section{Results}

For both models, the optimal emigration strategy included reverse tandem runs for a wide range of parameters, together with low quorum thresholds (Fig. 2). Fixing $k$ whilst varying $\mu$ and $F$, the optimal strategy often contained more reverse than forward tandem runs for a large part of the parameter range (Fig. 2). The fraction of time spent on reverse tandem running $f$ and the quorum threshold $Q$ were negatively correlated. When either the fraction of active ants $F$ decreased or the scouting parameter $\mu$ increased, fraction $f$ increased, and the quorum $Q$ decreased. Choosing a higher recruitment latency by decreasing $k$ gave more reverse tandem running and lower quorum thresholds (Fig. 2).

Note that, although models 1 and 2 broadly give similar predictions, they differ in the amount of postquorum time spent on reverse tandem runs. In model 1 , this reaches a full $100 \%$ in model 1 , but never so in model 2.

Overall, the models predict that reverse tandem running should be used more than forward tandem running, and the quorum threshold lowered, if the recruitment latency increases by decreasing $k$ (scouting ants wait longer before starting their first recruitment act), in combination with either a decreasing fraction of active ants $F$, or an increasing scouting parameter $\mu$. For all but very large $F$, the optimal quorum threshold corresponded to the time when all active ants have left the old nest to go scouting. In the absence of multiple new nests, the decision when to switch from forward tandem running to social carrying is thus best made at the old nest. Recruiters should thus apply the following rule: Continue forward tandem running until there are no ants left to perform them with and then switch to 
model 1
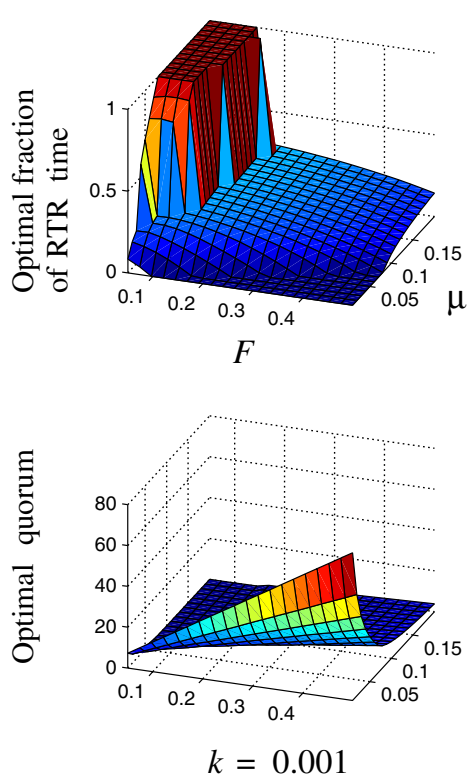

Fig. 2 Optimal fractions of post-quorum time spent on reverse tandem runs (top figures), and optimal quorum thresholds (bottom figures) for models 1 (left two columns) and 2 (right two columns) for varying fractions of active ants $F$ and scouting probabilities $\mu$ and for two values of recruitment latencies $k$.

social carrying; if few forward tandem runs have been performed (by the recruiters), combine carrying with reverse tandem runs; otherwise, do not.

The numbers of forward tandem runs, reverse tandem runs, the numbers of carried ants in the new nest and total emigration time were computed for each of the optimal strategies of models 1 and 2 (Fig. 3). We note four points. First, for a large parameter range, there are more reverse tandem runs than forward tandem runs. This is broadly consistent with the experimental data from nest choice experiments in Mallon et al. (2001). Numbers there range between 3 and 17 forward tandems, and between 9 and 25 reverse tandems, and reverse tandems were always performed more often than forward tandems. Second, as a validation of our optimisation method, note that the optimal emigration time varies smoothly under parameter changes, as is to be expected for this type of model. Third, for model 1, despite a clear drop in the post-quorum time spent on reverse tandem runs with increasing $F$ (see Fig. 2, top left), the number of reverse tandem runs in fact varies smoothly. Fourth, the number of carried ants that remained in the nest at the end of the emigration is clearly different between models 1 and 2 . In model 1 , this number is just $(1-F) N$, the number of passive ants in the colony. In model 2, however, over half of the colony may end up

model 2
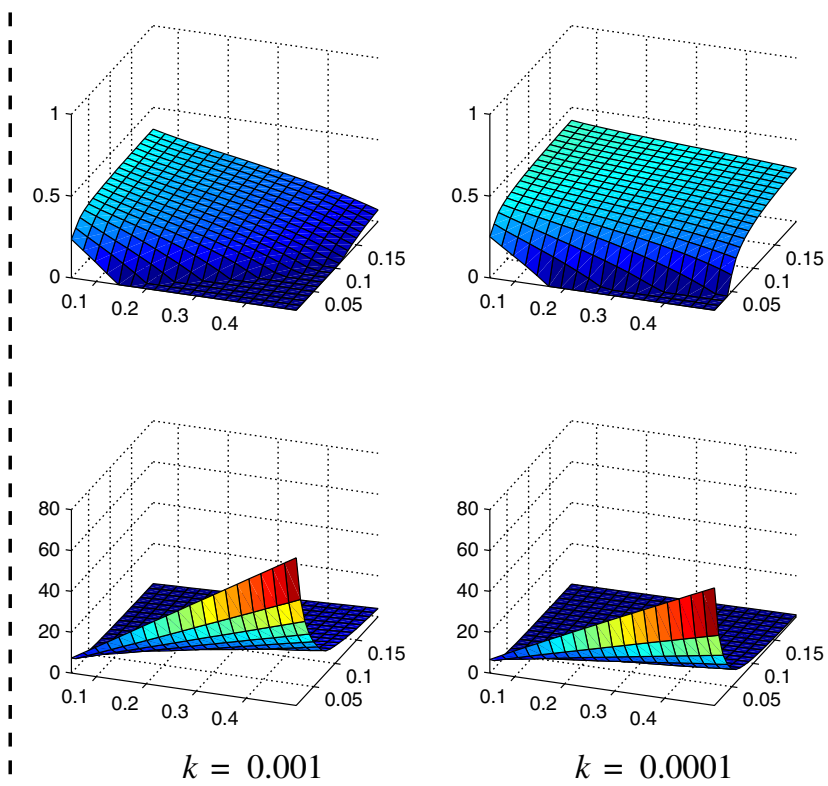

Other parameter values used are given in Table 1 . See text for simulation details. For both values of $k$, reverse tandem runs are part of the optimal emigration strategy when scouting probability is high or when fraction of active ants is low. Lowering $k$ enhances the use of reverse tandem running

being recruiters by drawing recruits from the carried class using reverse tandem runs.

\section{Discussion}

To maximise their fitness, ants should try to achieve the fastest emigrations to minimise vulnerability (Franks et al. 2003a,b). Therefore, the active ants either have to become scouts, discover a new site and then become recruiters or wait at the old nest until a recruiter leads them to the new nest. Both of these processes may be hampered: When all the active ants go out scouting, recruiter numbers slowly increase if the new nest is hard to find or if those few cannot find any active ant back at the old nest to tandem run with. In terms of the models, this could occur if scouts slowly become recruiters (low value for $k$ ), in combination with either a small class of active ants at the old nest ( $F$ is small) or all active ants having gone scouting (high value for scouting rate $\mu)$. Under either or both of these circumstances, the model predicts that ants should not waste time trying to recruit by forward tandem runs but should do the next best thing and use a low quorum threshold to quickly switch to carrying. The recruiters should then invest a fraction of their time to recruit scouts or carried ants using reverse tandem runs, thus boosting the recruiter population and speeding up the emigration. 

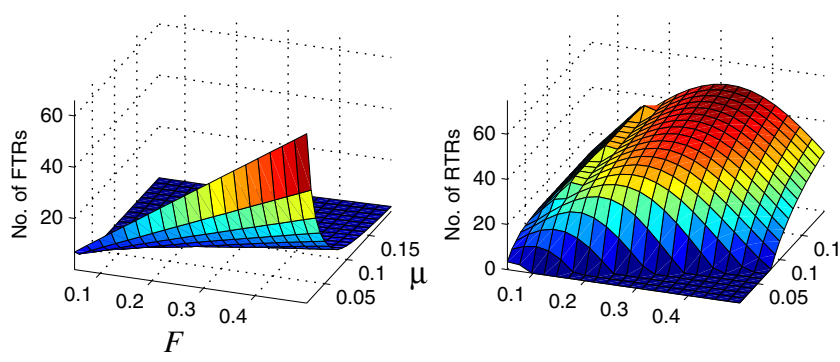

model 1
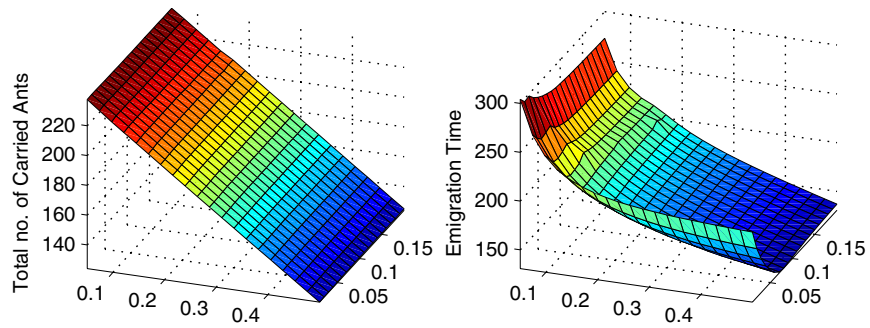

model 2
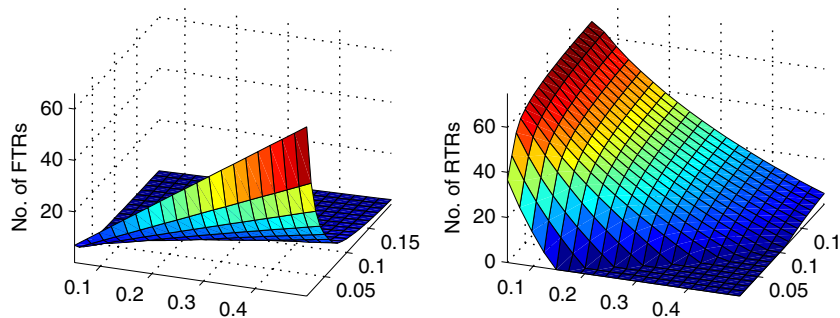

Fig. 3 Numbers of forward and reverse tandem runs, number of carried ants and emigration time, computed for each of the optimal strategies for models 1 (top row) and 2 (bottom row),

Conversely, the model also predicts that reverse tandem runs should not be used if either the new nest is easy to find (recruiter numbers then build quickly anyway), or when there are many ants to follow a forward tandem run.

These predictions fit quite well with previous experimental work. Ants have been shown to leave their intact old nest if the new nest is sufficiently better, but have lower standards when their nest is destroyed (Dornhaus et al. 2004). In these experiments, reverse tandem runs were mainly observed when the old nest was destroyed, combined with few forward tandem runs. The model offers a simple explanation for this: The greater panic might have caused fewer scouts to remain at the old nest, thereby obstructing early recruitment.

Whilst investigating speed-accuracy trade-offs, Franks et al. (2003a) found lower quorums under harsh than under mild conditions. This again fits with the models. On the other hand, the models also predict higher numbers of reverse tandem runs. In the experiments, this difference in reverse tandem running activity between mild and harsher conditions was found to be non-significant (Franks et al. 2003a).

\section{Critique on model 2}

Although evidence for which ants follow reverse tandem runs is scarce, both models give the same

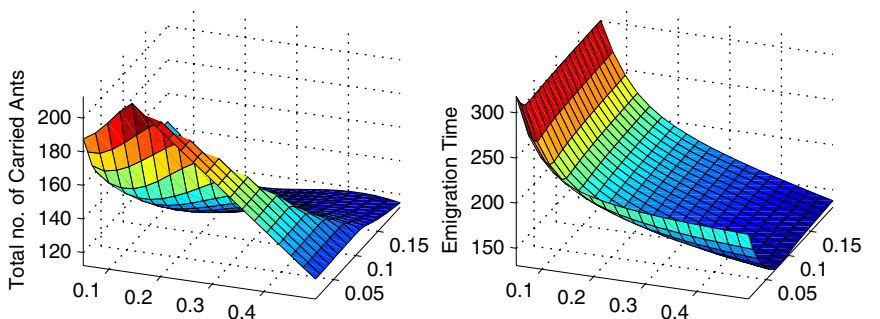

illustrated in Fig. 2. See that figure for details and parameter choices. Here we have only illustrated $k=0.001$

qualitative predictions. Note, however, that the dynamics of the different ant classes during a simulated emigration in model 2 poorly match observed experimental dynamics (see, e.g., Planqué et al. 2006). In many cases, the final number of ants active in an emigration is much greater than the original number of active ants. An example is given in Fig. 4. These recruiter numbers far exceed observed numbers of active ants (available in Table 3 in Pratt et al. 2005). Indeed, simulated emigrations often end with half the colony being recruiters and less than half carried into the new nest (Fig. 3, bottom row, third from left).

\section{Reverse tandem activity}

Figure 2 (top left) shows that recruiters in model 1 should use a sequential strategy if $F$ is small (and $\mu$ is large): When the quorum is met, they first spend all of their time on reverse tandem runs until all scouts have become recruiters, and then switch to carrying. In contrast, when $F$ is large, recruiters mix tandem running and carrying. This qualitative difference may be understood as follows.

The total number of recruiters is bounded by $F N$, the number of active ants in the colony. As $F$ decreases, the remaining recruiters take longer to carry all the passive ants. Hence, the time costs for not having the recruiters increases and the time to recruit the remaining scouts decreases (as there are fewer scouts too). 

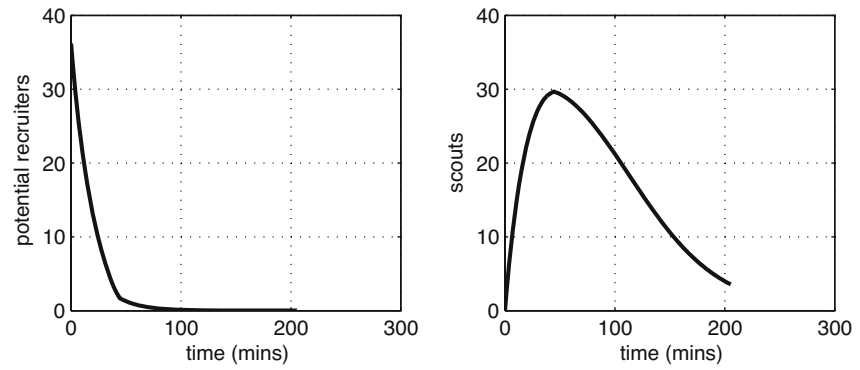

model 1
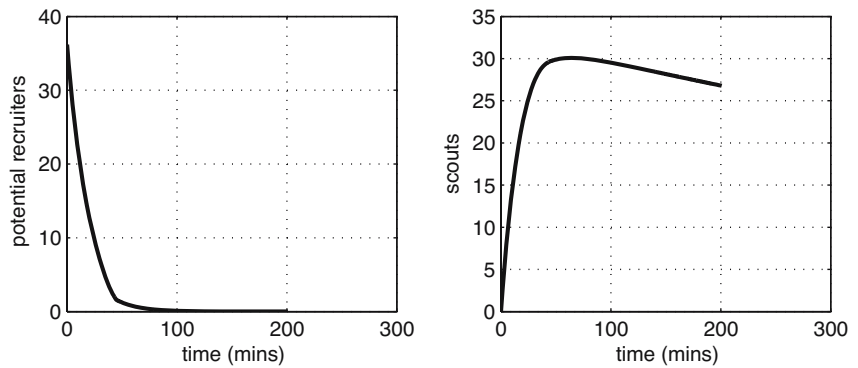

model 2
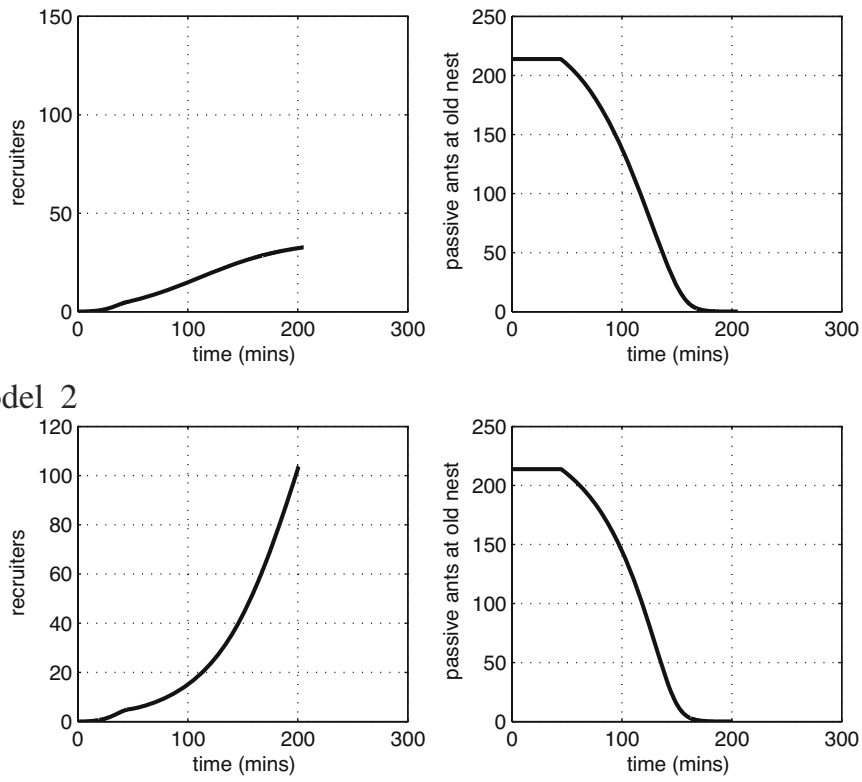

Fig. 4 Examples of temporal dynamics for models 1 and 2. At $\mu=0.05, F=0.1447$, we have taken parameters optimal for models 1 and 2, respectively. Notice that, in model 1, the number of recruiters rises to about 35 , but in model 2 , there are no less

than 100 recruiters at the end of the emigration, indicating that recruitment from the carried class (model 2) may give rise to very unrealistic emigration dynamics

Hence, in this situation, recruiters should devote their post-quorum time, first, all on reverse tandem running before starting carrying.

When $F$ is large, the reverse argument applies. With less passive ants, there should be less emphasis on additional recruitment by reverse tandem running. One does not have to make many hands if the work was light to start with.

Note that this behaviour for $F \rightarrow 0$ is different for model 2. In this paper, we never observe sequential strategies (Fig. 2, top right), as there is no end to building recruiter numbers but by completing the entire emigration.

This argument also explains another difference between these models: The number of reverse tandem runs during an emigration. In model 1 , there is a clear maximum for intermediate $F$, whereas in model 2 the number of reverse tandems strictly decreases with $F$ (Fig. 3, second from left, top and bottom).

Nonlinearities in the models and divisions between active and passive ants

Contrary to the models in this paper, two previous models of house hunting by T. albipennis ants (Pratt et al. 2002; Planqué et al. 2006) assumed linear terms for tandem running and social carrying. There, these processes occurred at rates only proportional to the number

of recruiters. The predictions of the current models proved to be strongly dependent on the assumption of non-linearity of these terms. The corresponding linear models predicted that reverse tandem running should not be used for practically any parameter choices in $F$ and $\mu$ or $k$.

Another ingredient in this model shared with the previous (Pratt et al. 2002; Planqué et al. 2006), models of this collective decision-making system is the division between active and passive ants. There is as yet little experimental evidence suggesting this division really exists. All we know at present is that a limited fraction of ants is actively engaged during an emigration. We have thus also explored models in which this division was absent, using both linear and non-linear interaction terms such as those in models 1 and 2 presented in this paper. In none of these models did reverse tandem running contribute to the optimal emigration speed. The division between active and passive ants is thus a crucial ingredient for reverse tandem running to have a positive impact on emigration speed, which should be experimentally validated.

\section{Hypothesised explanations}

Several hypotheses on the potential role of reverse tandem running have been put forward (Pratt et al. 2002). First, the ants might have a "home nest", which changes 
during the emigration, thereby reversing the direction of any recruitment events from "home" to another nest (Pratt et al. 2002). If true, this would predict a change of direction when about half of the colony had been displaced. This is not in agreement with the available data (Mallon et al. 2001). Moreover, this hypothesis does not offer a suggestion why tandem running often occurs early in the emigration. In other words, it might explain the direction, but not the occurrence itself.

Second, it has been suggested that reverse tandem running may re-allocate recruitment (Pratt et al. 2002). Again, this does not fit the available data from Mallon et al. (2001). Reverse tandem runs were nearly always observed between the best nest and the old nest. The models in this paper do not incorporate choice between nest sites, but we conjecture that early flexible commitment (Planqué et al. 2006) will be more efficient in redirecting ants to better nests than late recruitment. Other experimental results also corroborate that reverse tandem running does not influence the decisionmaking process (Franks et al. 2003a).

The first models in which reverse tandem runs have been explicitly incorporated to analyse their role have yielded clear predictions: under a range of conditions, we expect a negative correlation between levels of early and late recruitment. This finding lends itself well to simple experiments, and we aim to present those in the near future.

The build up of recruiter numbers serves two purposes: to decide on a nest and to increase the number of ants actively involved in transport. The decisionmaking process and the implementation of this decision are thus conflated. This in itself is a side-effect of the distributed nature of this system. Reverse tandem running may thus be a logical extension to overcome this inherent problem. This suggests that such additional backup behaviours could be a common feature of decentralised collective decision-making systems.

Acknowledgements We would like to thank all the members of the Bristol Ant Lab and two anonymous referees for their insightful comments and ideas. RP, NRF, JARM and TK are pleased to acknowledge the support of an EPSRC grant (GR/S78674/01). RP was also kindly supported by the NDNS+ cluster, financed by the National Science Foundation NWO. NRF and FXDM are grateful for the support of a BBSRC grant (E19832).

\section{References}

Able K, Bingman V (1987) The development of orientation and navigation behavior in birds. Q Rev Biol 62:1-29

Britton N, Planqué R, Franks N (2007) Evolution of defence portfolios in exploiter-victim system. Bull Math Biol 69(3):957-88
Davies N (2000) Cuckoos, cowbirds and other cheats. T. \& A.D. Poyser, London

Dornhaus A, Franks N, Hawkins R, Shere H (2004) Ants move to improve-colonies of Leptothorax albipennis emigrate whenever they find a superior nest site. Anim Behav 67: 959-963

Franks N, Dornhaus A, Fitzsimmons J, Stevens M (2003a) Speed versus accuracy in collective decision making. Proc R Soc Lond B 270:2457-2463

Franks N, Mallon E, Bray H, Hamilton M, Mischler T (2003b) Strategies for choosing between alternatives with different attributes: exemplified by house-hunting ants. Anim Behav 65:215-223

Franks N, Richardson T (2006) Teaching in tandem-running ants. Nature 439:153

Langridge E (2006) The effects of experience on complete ant colonies. PhD thesis, University of Bristol.

Mallon E, Franks N (2000) Ants estimate area using Buffon's needle. Proc R Soc Lond B 267:765-770

Mallon E, Pratt S, Franks N (2001) Individual and collective decision-making during the nest site selection by the ant Leptothorax albipennis. Behav Ecol Sociobiol 50: 352-359.

Marshall J, Dornhaus A, Franks N, Kovacs T (2006) Noise, cost and speed-accuracy trade-offs: decision making in a decentralised system. J R Soc Interface, 3:243-254.

Möglich M (1978) Social organization of nest emigration in Leptothorax (Hym., Form.). Insectes Soc 25(3):205-225

Möglich M, Maschwitz U, Hölldobler B (1974) Tandem calling: a new kind of signal in ant communication. Science 186: 1046-1047

Nelder J, Mead R (1965) A simplex method for function minimization. Comput J 7:308-313

Perelson A (2002) Modelling viral and immune systems. Nat Rev Immunol 2:28-36

Planqué R, Britton N, Franks N, Peletier M (2002) The adaptiveness of defence strategies against cuckoo parasitism. Bull Math Biol 64(6):1045-1068

Planqué R, Dornhaus A, Franks N, Kovacs T, Marshall J (2006) Weighting waiting in collective decision making. Behav Ecol Sociobiol 61(3):347-356

Pratt S (2005) Quorum sensing by encounter rates in the ant Temnothorax albipennis. Behav Ecol 16(2):488-496

Pratt S, Mallon E, Sumpter D, Franks N (2002) Quorumdependent recruitment and collective decision-making during colony emigration by the ant Leptothorax albipennis. Behav Ecol Sociobiol 52:117-127

Pratt S, Sumpter D, Mallon E, Franks N (2005) An agent-based model of collective nest choice by the ant Leptothorax albipennis. Anim Behav 70:1023-1036

Rehr S, Feeny D, Janzen D (1973) Chemical defense in Central American non-ant acacias. J Anim Ecol 42:405-416

Seeley T (1977) Measurement of nest cavity volume by the honey bee (Apis mellifera). Behav Ecol Sociobiol 2:201-227

Seeley T, Morse R (1978) Nest site selection by the honey bee, Apis mellifera. Insectes Soc 25(4):323-337

Seeley T, Visscher P (2003) Choosing a home: how the scouts in a honeybee swarm perceive the completion of their group decision making. Behav Ecol Sociobiol 54:511-520

Seeley T, Visscher P (2004) Quorum sensing during nest-site selection by honeybee swarms. Behav Ecol Sociobiol 56: 594-601

Visscher P (2007) Group decision making in nest-site selection among social insects. Annu Rev Entomol 52:255-275

Wiltschko R, Wiltschko W (2003) Avian navigation: from historical to modern concepts. Anim Behav 65:257-272 